\title{
Resveratrol inhibits decidualization by accelerating downregulation of the CRABP2-RAR pathway in differentiating human endometrial stromal cells
}

\author{
Asako Ochiai ${ }^{1}$, Keiji Kuroda ${ }^{1,2}$, Rie Ozaki ${ }^{1}$, Yuko Ikemoto ${ }^{1}$, Keisuke Murakamii ${ }^{1}$, Joanne Muter ${ }^{3}$, Akemi Matsumoto ${ }^{1}$, \\ Atsuo Itakura', Jan J. Brosens (10,4 and Satoru Takeda'
}

\begin{abstract}
Pregnancy critically depends on the transformation of the human endometrium into a decidual matrix that controls embryo implantation and placenta formation, a process driven foremost by differentiation and polarization of endometrial stromal cells into mature and senescent decidual cells. Perturbations in the decidual process underpin a spectrum of prevalent reproductive disorders, including implantation failure and early pregnancy loss, emphasizing the need for new therapeutic interventions. Resveratrol is a naturally occurring polyphenol, widely used for its antioxidant and anti-inflammatory properties. Using primary human endometrial stromal cell (HESC) cultures, we demonstrate that resveratrol has anti-deciduogenic properties, repressing not only the induction of the decidual marker genes PRL and IGFBPI but also abrogating decidual senescence. Knockdown of Sirtuin 1, a histone deacetylase activated by resveratrol, restored the expression of IGFBPI but not the induction of PRL or senescence markers in decidualizing HESCs, suggesting involvement of other pathways. We demonstrate that resveratrol interferes with the reprogramming of the retinoic acid signaling pathway in decidualizing HESCS by accelerating down-regulation of cellular retinoic acid-binding protein 2 (CRABP2) and retinoic acid receptor (RAR). Notably, knockdown of CRABP2 or RAR in HESCs was sufficient to recapitulate the anti-deciduogenic effects of resveratrol. Thus, while resveratrol has been advanced as a potential fertility drug, our results indicate it may have detrimental effects on embryo implantation by interfering with decidual remodeling of the endometrium.
\end{abstract}

\section{Introduction}

Resveratrol (3,5,4'-trihydroxystilbene), a natural polyphenolic compound found in grapes, nuts, and berries, is widely studied because of its antioxidative, anti-inflammatory and insulin-sensitizing properties ${ }^{1,2}$. It is a potent activator of Sirtuin 1 (SIRT1), a nicotinamide adenine dinucleotide (NAD)-dependent histone

\footnotetext{
Correspondence: Keiji Kuroda (kuroda@sugiyama.or.jp)

${ }^{1}$ Department of Obstetrics and Gynaecology, Faculty of Medicine, Juntendo

University, Tokyo 113-8421, Japan

${ }^{2}$ Center for Reproductive Medicine and Implantation Research, Sugiyama Clinic

Shinjuku, Tokyo 116-0023, Japan

Full list of author information is available at the end of the article.

Edited by E. Candi
}

deacetylase that targets numerous transcription factors involved in metabolic homeostasis, cell differentiation, apoptosis and senescence ${ }^{3-5}$. Resveratrol has been mooted as a potentially therapeutic agent for infertile patients with diminished ovarian reserve, obesity, and polycystic ovary syndrome $(\mathrm{PCOS})^{6-10}$. In addition, a number of studies pointed towards the therapeutic potential of resveratrol in improving testicular function and sperm quality as well as in the management of pelvic endometriosis and uterine leiomyomas ${ }^{11-13}$. However, the potential impact of resveratrol on endometrial preparation for embryo implantation has not yet been evaluated. 
During the mid-luteal phase, the human endometrium starts to remodel intensely, heralding a transient window for embryo implantation. This implantation window coincides with the differentiation of endometrial stromal cells into specialized decidual cells. In pregnancy, tightly adherent decidual cells form a nutritive matrix around the early conceptus that controls trophoblast invasion and regulates maternal immune tolerance of the antigenicallydistinct fetus ${ }^{14}$. A recent study demonstrated that human endometrial stromal cells (HESCs) polarize upon differentiation into mature and acutely senescent decidual subpopulations ${ }^{15}$. Interleukin-15 (IL-15) activated uterine

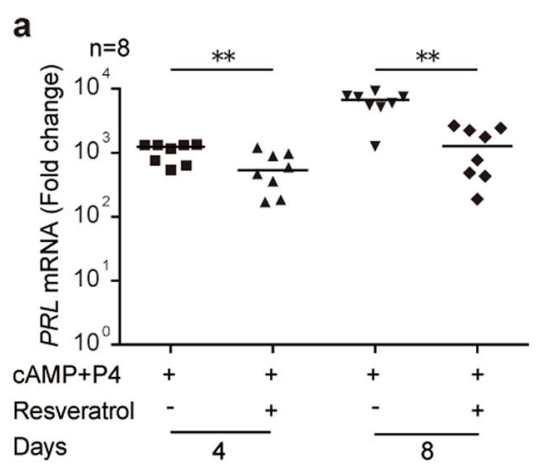

b

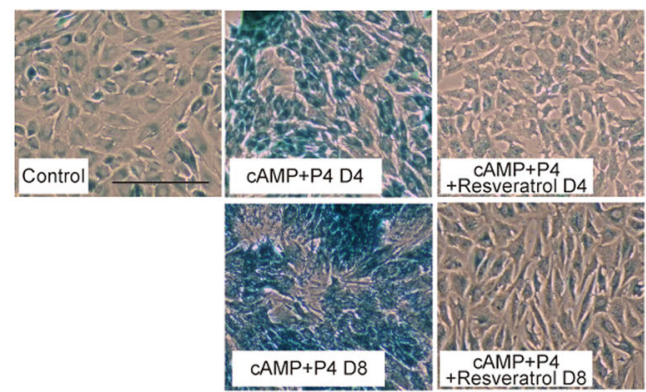

C

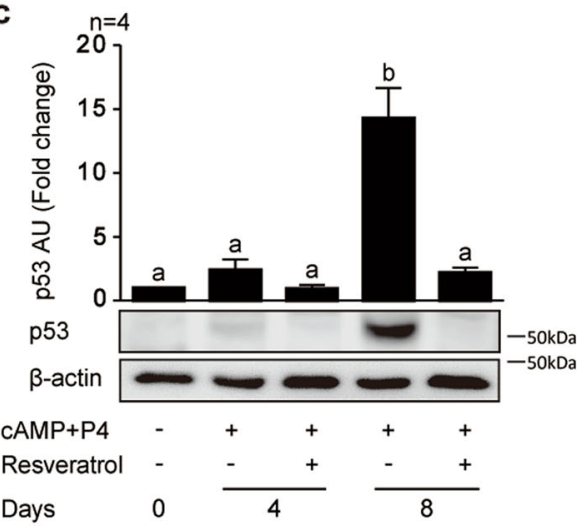

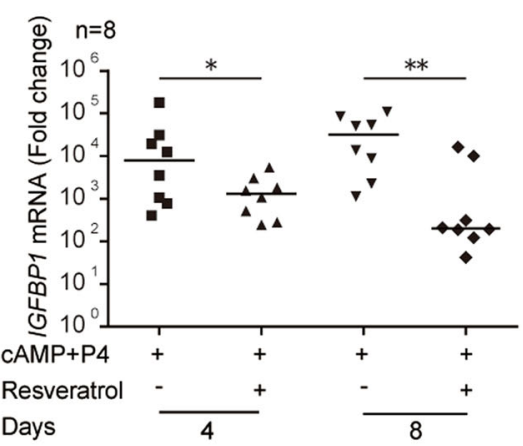

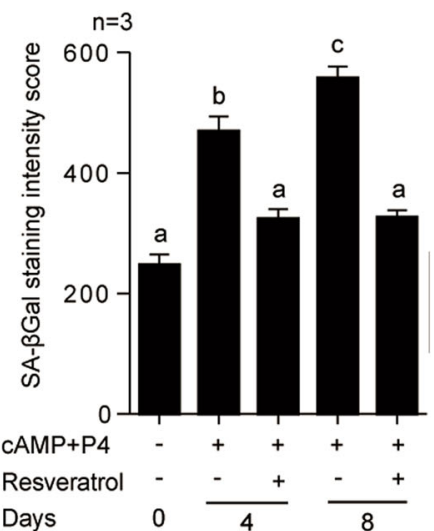

d

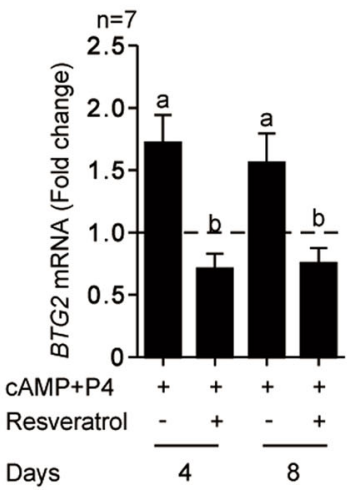

Fig. 1 Resveratrol inhibits decidualization a RTQ-PCR analysis of PRL and IGFBP1 transcript levels in primary HESC cultures $(n=8)$ treated with CAMP and P4 in combination with or without resveratrol $(100 \mu \mathrm{M})$ for 4 or 8 days. ${ }^{*} P<0.05$; ${ }^{* *} P<0.01$. b Representative senescence-associated $\beta$ galactosidase $(S A B G)$ staining of parallel cultures. Original magnification: $\times 100$. Scale bar: $200 \mu m$ (left panel). SABG staining intensity score calculated from 300 cells in three random fields. Data show fold-change (mean \pm SEM) relative to vehicle control (right panel). c Representative Western blot analysis and quantification of p53 levels in whole-cell lysates from 4 independent cultures decidualized in the presence or absence of resveratrol for the indicated timepoints. $\beta$-actin serves as a loading control. d RTQ-PCR analysis of BTG2 transcript levels in primary HESCS treated as indicated $(n=7)$. Data show fold-change (mean \pm SEM) relative to vehicle-treated undifferentiated cells (dotted line). Different letters above error bars denote significant difference at $P<0.05$ 
natural killer (uNK) cells then target and eliminate senescent decidual cells via granule exocytosis, enabling the cycling endometrium to transition into a gestational tissue upon embryo implantation ${ }^{15}$.

Differentiation of HESCs into mature decidual cells critically depends on coordinated reprogramming of multiple signaling pathways ${ }^{16-19}$, including the retinoic acid (RA) pathway ${ }^{20,21}$. The cellular responses to RA are mediated by distinct nuclear receptors; RA receptors (RAR) and peroxisome proliferator activated receptors (PPAR) $\beta / \delta$, which promote apoptosis and cellular differentiation, respectively ${ }^{22-24}$. The nature of the cellular response to RA signaling is regulated by two intracellular RA-binding proteins, cellular retinoic acid-binding protein 2 (CRABP2) and fatty acid-binding protein 5 (FABP5). RA bound to CRABP2 in the cytoplasm activates the RAR, which leads to hetero-dimerization with retinoid $\mathrm{X}$ receptor (RXR) and activation of genes associated with cell cycle arrest and apoptotic machinery. By contrast, binding of RA to FABP5 activates PPAR $\beta / \delta$, which induces cellular differentiation. Upon decidualization, HESCs downregulate CRABP2 and, to a lesser extent, FABP5. RAR is also downregulated whereas $\operatorname{PPAR} \beta / \delta$, which transduces the differentiation responses of RA, is induced ${ }^{20}$.

SIRT1 is an important modulator of RA signaling. For example, SIRT1 interacts with and deacetylates CRABP2, which sequesters this RA binding protein in the cytoplasm $^{25}$. SIRT1 also suppresses the transcriptional activity of $\mathrm{RAR}^{26,27}$. In light of these observations, we hypothesized that resveratrol-mediated SIRT1 activation in HESCs would favor the FABP5-PPAR $\beta / \delta$ pathway, thereby promoting decidualization. Unexpectedly, we observed that exposure of primary culture to resveratrol blocks subsequent differentiation of HESCs into mature and senescent cells by accelerating downregulation of CRABP2-RAR pathway.

\section{Results}

\section{Resveratrol suppresses decidualization}

An initial dose-response experiment indicated that 100 $\mu \mathrm{M}$ of resveratrol significantly inhibited the induction of PRL (encoding prolactin), a widely used decidual marker gene, in primary cultures treated with 8-bromoadenosine $3^{\prime} 5^{\prime}$-cyclic AMP (cAMP) and progesterone (P4) for 4 days (Supplementary Figure S1). To validate this observation, 8 independent primary cultures were decidualized with cAMP and P4 for either 4 or 8 days in the presence or absence of $100 \mu \mathrm{M}$ of resveratrol. As shown in Fig. 1a, resveratrol not only inhibited the induction of $P R L$ but also attenuated IGFBP1 (coding insulin-like growth factor-binding protein-1) expression in decidualizing cultures. By contrast, pre-treatment of primary cultures with $100 \mu \mathrm{M}$ resveratrol for $48 \mathrm{~h}$ followed by wash-off and decidualization in the absence of resveratrol had no impact on the induction of either $P R L$ and IGFBPI (Supplementary Figure S2). Thus, the anti-deciduogenic actions of resveratrol are temporally confined to the process of active differentiation.

Recent studies demonstrated that primary HESCs polarize into two subpopulations upon decidualization, representing mature and acutely senescent decidual cells $^{15,28}$. To examine the impact of resveratrol on decidual senescence, primary cultures differentiated for either 4 or 8 days in the presence or absence resveratrol were stained for senescence-associated $\beta$-galactosidase (SABG) activity, a widely used biomarker for senescent cells $^{29}$ (Fig. 1b). As expected, decidualization was associated with a time-dependent increase in $\mathrm{SA}_{\beta} \mathrm{G}^{+}$cells. Resveratrol abolished the induction $\mathrm{SA} \mathrm{G}^{+}$cells in primary HESCs treated with cAMP and P4 (Fig. 1b). To substantiate this observation, we examined the levels of the tumor suppressor protein p53, a critical mediator of decidual senescence ${ }^{15,30}$, in primary cultures decidualized in the presence or absence of resveratrol. Again, resveratrol abrogated p53 accumulation in differentiating HESCs (Fig. 1c). We also examined the induction of B cell translocation gene 2 (BTG2), an anti-proliferation gene induced by RA in MCF-7 breast cancer cells ${ }^{31,32}$. BTG2 expression was upregulated upon decidualization, in keeping with the need for HESCs to exit the cell cycle prior to differentiation ${ }^{15}$, and again this response was blocked by resveratrol (Fig. 1d). Taken together, the data show that resveratrol is a potent inhibitor of decidualization, including decidual senescence.

\section{SIRT1 knockdown does not reverse resveratrol-mediated decidual repression}

To determine the mechanism of resveratrol action in differentiating HESCs, we first profiled the expression of SIRT1, a NAD-dependent deacetylase activated by resveratrol, in primary cultures differentiated for 4 and 8 days in the presence or absence of resveratrol treatment. As shown in Fig. 2a, SIRT1 transcript levels were unchanged after 4 days of decidualization with cAMP and P4 but down-regulated by day 8. By contrast, decidualization in the presence of resveratrol resulted in significant increase in SIRT1 mRNA levels at both timepoints $(P<0.01)$. Western blot analysis showed that downregulation of SIRT1 in decidualizing cells was more pronounced at protein level when compared to mRNA level (Fig. 2b), potentially indicating a posttranscriptional mechanism of regulation. Nevertheless, resveratrol reversed the downregulation of SIRT1 in response to cAMP and $\mathrm{P} 4$ treatment resulted in significantly higher SIRT1 levels by day 8 of decidualization $(P<0.05)$. Immunohistochemistry confirmed nuclear accumulation of SIRT1 upon decidualization in the presence of 
a

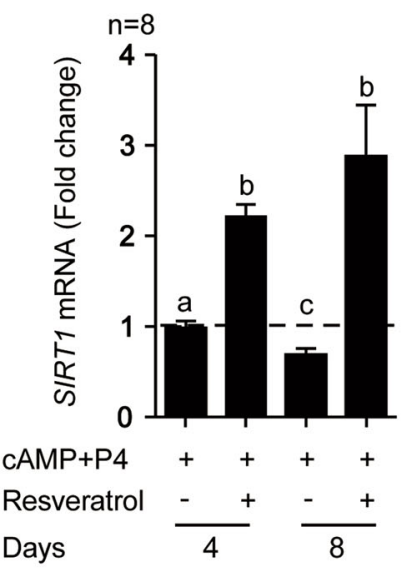

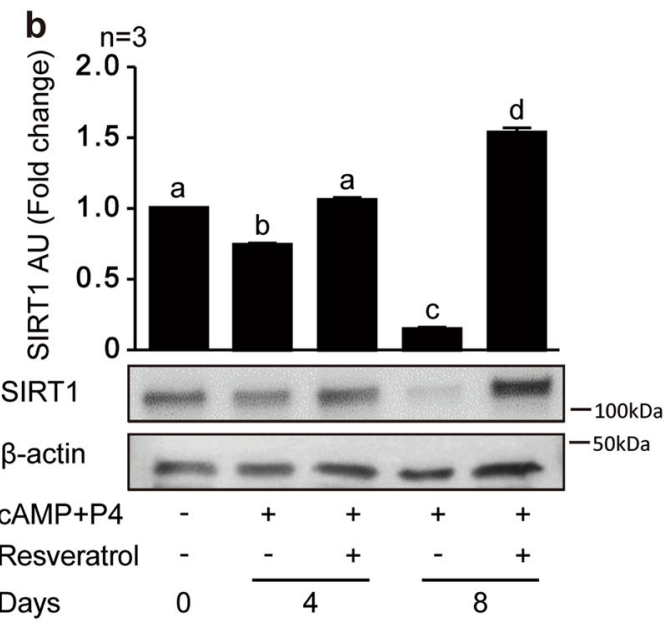

Days

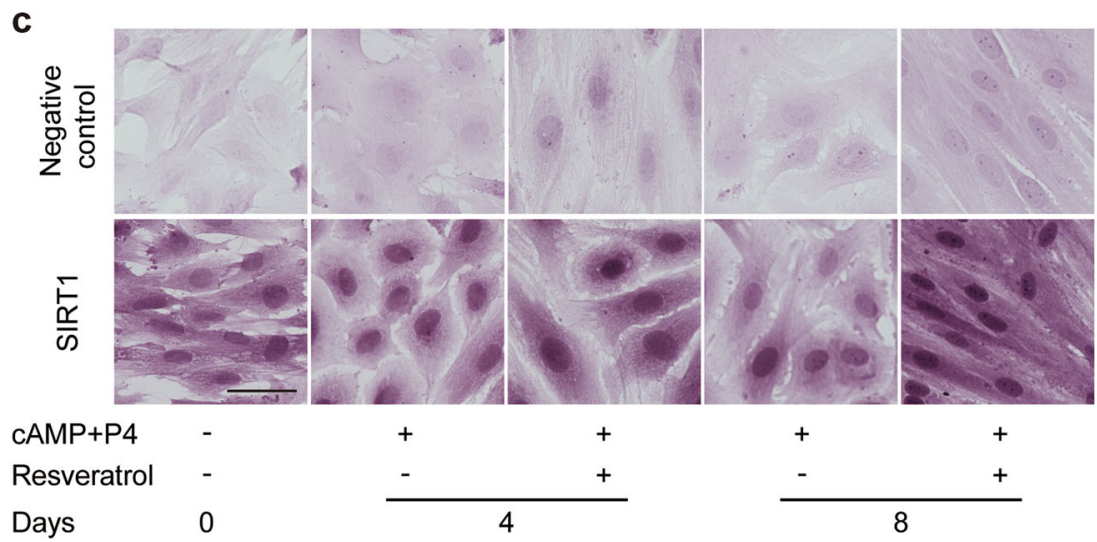

Fig. 2 Resveratrol regulates SIRT1 expression in decidualizing HESCs. a SIRT1 mRNA levels in undifferentiated and decidualized cells treated with CAMP and P4 in combination with or without resveratrol $(100 \mu \mathrm{M})$ for 4 or 8 days. Data show fold-change (mean \pm SEM) relative to undecidualized vehicle control (dotted line) of 8 independent experiments. b Representative Western blot and quantification of SIRT1 levels in whole cell lysates from 3 independent cultures treated in parallel. $\beta$-actin serves as a loading control. Different letters above error bars denote significance at $P<0.05$. $\mathbf{c}$ Representative immunohistochemical images of SIRT1 expression in HESC cultures treated as indicated. Original magnification: $\times 200$. Scale bar: $50 \mu \mathrm{m}$

resveratrol for 4 or 8 days. Further, acquisition of the typical decidual morphology, characterized by nuclear and cytoplasmic enlargement, was blocked in the presence of resveratrol with cells retaining a fibroblastic appearance (Fig. 2c).

To determine if SIRT1 activation was responsible for the anti-deciduogenic actions of resveratrol, primary cultures were first transfected with either non-targeting (NT) siRNA or siRNA targeting SIRT1 and then decidualized with cAMP and P4 in the presence or absence of resveratrol for 4 days. The knockdown efficacy was high with SIRT1 mRNA level in resveratrol treated cultures remaining below that observed in untreated control cells (Fig. 3a, left panel). Interestingly, SIRT1 knockdown had no effect on resveratrol-mediated inhibition of $P R L$ expression (Fig. 3a, middle panel), whereas IGFBP1 expression was restored (Fig. 3a, right panel). Further,
SIRT1 knockdown did not restore decidual senescence in resveratrol-treated cultures as ascertained by SA $\beta G$ (Fig. 3b). To corroborate this observation, total protein lysates of cultures first transfected with either NT or SIRT1 siRNA and then decidualized for 4 days in the presence or absence of resveratrol were subjected to Western blot analysis. As shown in Fig. 3c, SIRT1 knockdown increased p53 accumulation in decidualizing cultures, although downregulation of p53 in response to resveratrol treatment was not reversed. Furthermore, SIRT1 knockdown was also insufficient to reverse resveratrol-mediated downregulation of BTG2 mRNA levels in decidualizing cultures (Fig. 3d). Taken together, the data indicate that SIRT1 activation in response to resveratrol exposure of decidual cells accounts for repression of selective decidual genes, such as IGFBP1. However, resveratrol-mediated repression of other 
a

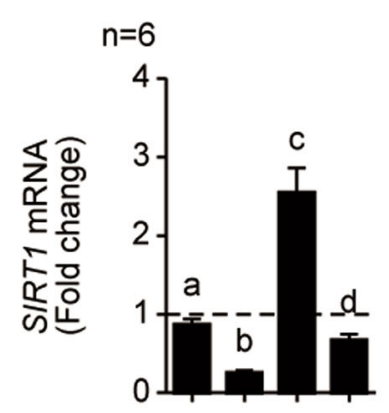

CAMP+P4

NT SiRNA

SIRT1 SiRNA - + + +

Resveratrol - - + +

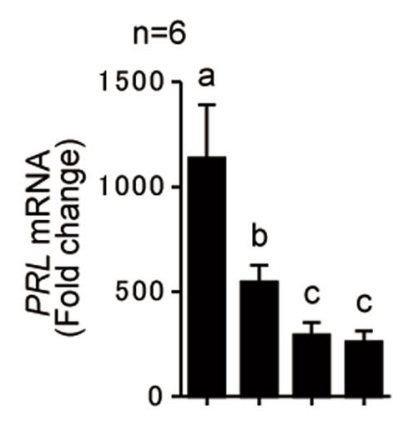

CAMP+P4

NT SiRNA

SIRT1 SiRNA - + - +

Resveratrol - - + +

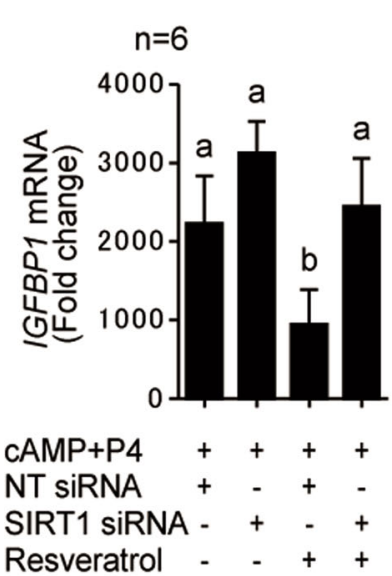

b

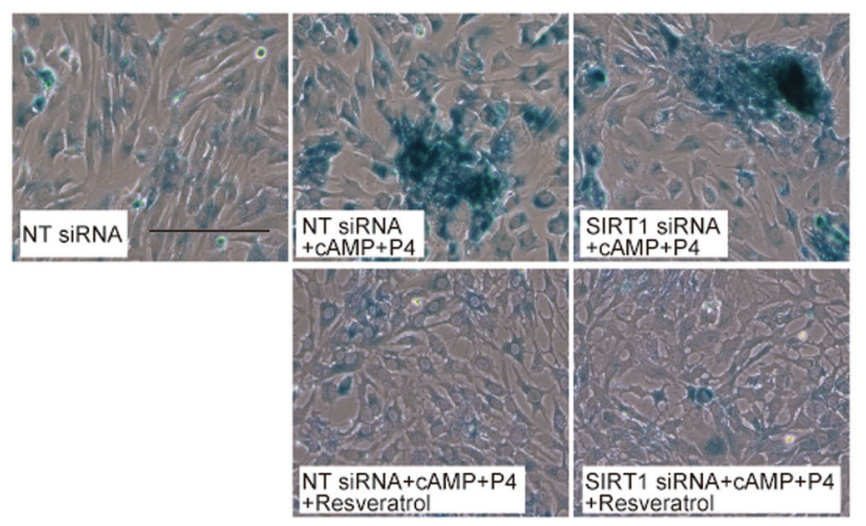

C

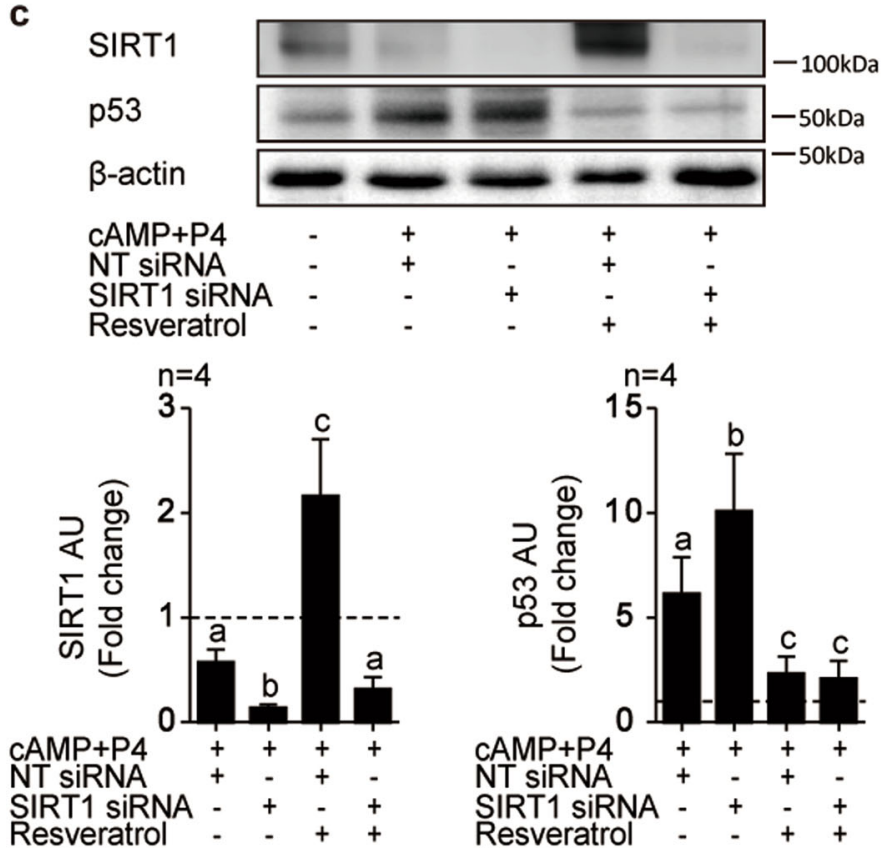

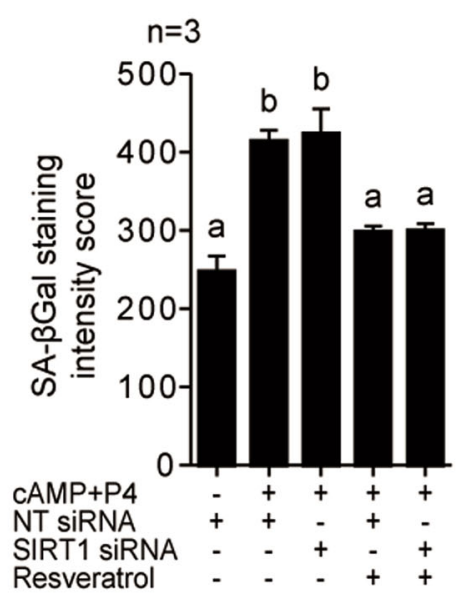

d

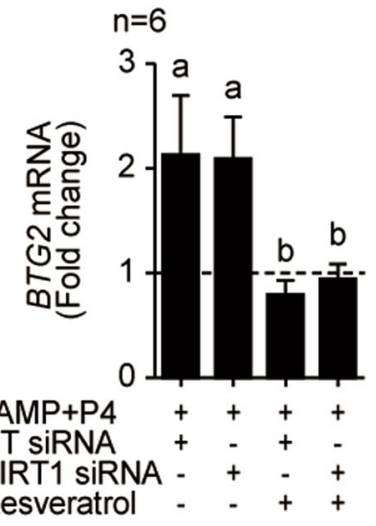

Fig. 3 (See legend on next page.) 
(see figure on previous page)

Fig. 3 Impact of SIRT1 knockdown on resveratrol actions in decidualizing HESCs. a RTQ-PCR analysis of SIRT1, PRL, and IGFBP1 transcript levels in HESC cultures first transfected with NT or SIRT1 siRNAs and then treated with CAMP and P4 in combination with or without resveratrol for 4 days. The experiment was carried out in 6 independent primary cultures. b Representative SABG staining of parallel HESC cultures. Original magnification: $\times 100$. Scale bar: $200 \mu \mathrm{m}$. SAßG staining intensity score was calculated from 300 cells in 3 random fields (right panel) and data show mean \pm SEM score of 3 independent cultures. c Representative Western blot and quantification of SIRT1 and p53 proteins in whole-cell lysates obtained from parallel HESC cultures $(n=4)$. $\beta$-actin serves as a loading control. d RTQ-PCR analysis of BTG2 transcript levels in 6 independent primary cultures treated as indicated. Data show fold-change (mean \pm SEM) relative to vehicle-treated undifferentiated cells (dotted line). Different letters above error bars denote significance at $P<0.05$

decidual features, including p53 accumulation and cellular senescence, are SIRT1-independent.

\section{Resveratrol accelerates downregulation of the CRABP2- RARa pathway in decidualizing cells}

In other cell systems, resveratrol has been shown to modulate a number of signal transduction pathways, including RA signaling ${ }^{26,33}$. Decidual transformation of HESCs is accompanied with suppression of CRABP2RAR $\alpha$ signaling and induction of PPAR $\beta / \delta$, involved in cellular differentiation ${ }^{20}$. To monitor the impact of resveratrol on this pathway, total protein lysates from primary HESC cultures, decidualized for 1,2 , or 4 days in the presence or absence of resveratrol, were harvested for Western blot analysis. As expected, differentiation of HESCs with cAMP and P4 resulted in a time-dependent downregulation of CRABP2 and RAR $\alpha$ expression and concomitant rise in PPAR $\beta / \delta$ levels (Fig. 4a). Interestingly, resveratrol attenuated the induction of $\operatorname{PPAR} \beta / \delta$ and accelerated the downregulation CRABP2 and RAR $\alpha$, a response that was already apparent after $24 \mathrm{~h}$ of decidualization and maintained over 4 days.

Next, we performed additional real-time quantitative (RTQ)-PCR and Western blot analyses to determine if the effect of resveratrol of RA signal transduction pathway was SIRT1-dependent. As shown in Fig. 4b, c, SIRT1 knockdown had no impact on CRABP2 and RAR $\alpha$ expression in decidualizing cells, irrespectively of resveratrol treatment. By contrast, SIRT1 knockdown was sufficient to reverse resveratrol-mediated inhibition of $\operatorname{PPAR} \beta / \delta$ at both mRNA and protein level. The data further confirm that resveratrol impacts on decidual gene expression through SIRT1-dependent and SIRT1independent mechanism.

\section{Accelerated downregulation of the CRABP2-RARa pathway impairs decidualization}

Previous studies reported that differentiation of HESCs into decidual cells is a multi-step process, dependent on coordinated down- and up-regulation of distinct gene networks ${ }^{17,18,21}$. Hence, we speculated that accelerated downregulation of the CRABP2-RAR $\alpha$ pathway in response to resveratrol may be sufficient to disrupt the subsequent decidual phenotype. To test this hypothesis, three independent primary HESCs were first transfected with NT siRNA or siRNA targeting CRABP2 or RAR $\alpha$ and then decidualized with cAMP and P4 (Fig. 5a, b). When compared to undifferentiated cells, CRABP2 mRNA levels were reduced by 60 and $90 \%$ in cells decidualized for 4 days following transfected with NT or CRABP2 siRNA, respectively. CRABP2 knockdown had no impact on PPAR $\beta / \delta$ expression but repressed the induction of $P R L$ and IGFBP1 in decidualizing cells (Fig. 5a). Knockdown of RAR $\alpha$ recapitulated these findings (Fig. 5b). Notably, GW501516, a PPAR $\beta / \delta$ agonist, further enhanced the expression of its target nuclear receptors in cells decidualized for 4 days, but had no impact on either PRL or IGFBP1 mRNA levels (Supplementary Figure S3). Taken together, the data indicate that accelerated downregulation of the CRABP2-RAR $\alpha$ pathway blunts the induction of decidual marker genes in HESCs.

Finally, we investigated if coordinated downregulation of the CRABP2-RAR $\alpha$ pathway in differentiating HESCs is required for the emergence of senescent decidual cells. As shown in Fig. 5c, knockdown of CRABP2 or RAR $\alpha$ was sufficient to attenuate the induction of SA $\beta$ G in primary cultures treated with cAMP and $\mathrm{P} 4$ for 4 days. As expected, knockdown of CRABP2 or RAR $\alpha$ also inhibited p53 accumulation and induction of BTG2 expression in decidual cells (Fig. 5d, e).

\section{Discussion}

A limited window of implantation synchronizes embryo implantation in an optimal uterine environment. In mice, a postovulatory oestradiol surge both activates dormant embryos and renders the progesterone-primed endometrium receptive ${ }^{34}$. Recent evidence suggests that an acute auto-inflammatory endometrial response has replaced the obligatory maternal implantation signal in mice $^{15}$. In human endometrium, this inflammatory response associated with the window of implantation is thought to polarize HESCs into two decidual subpopulations ${ }^{15}$, through an evolutionarily conserved mechanism ${ }^{35}$. The first subpopulation consists of mature decidual cells, which will form a semi-permanent, nutritive matrix that controls trophoblast invasion and placental formation in pregnancy. The second subpopulation, however, are 

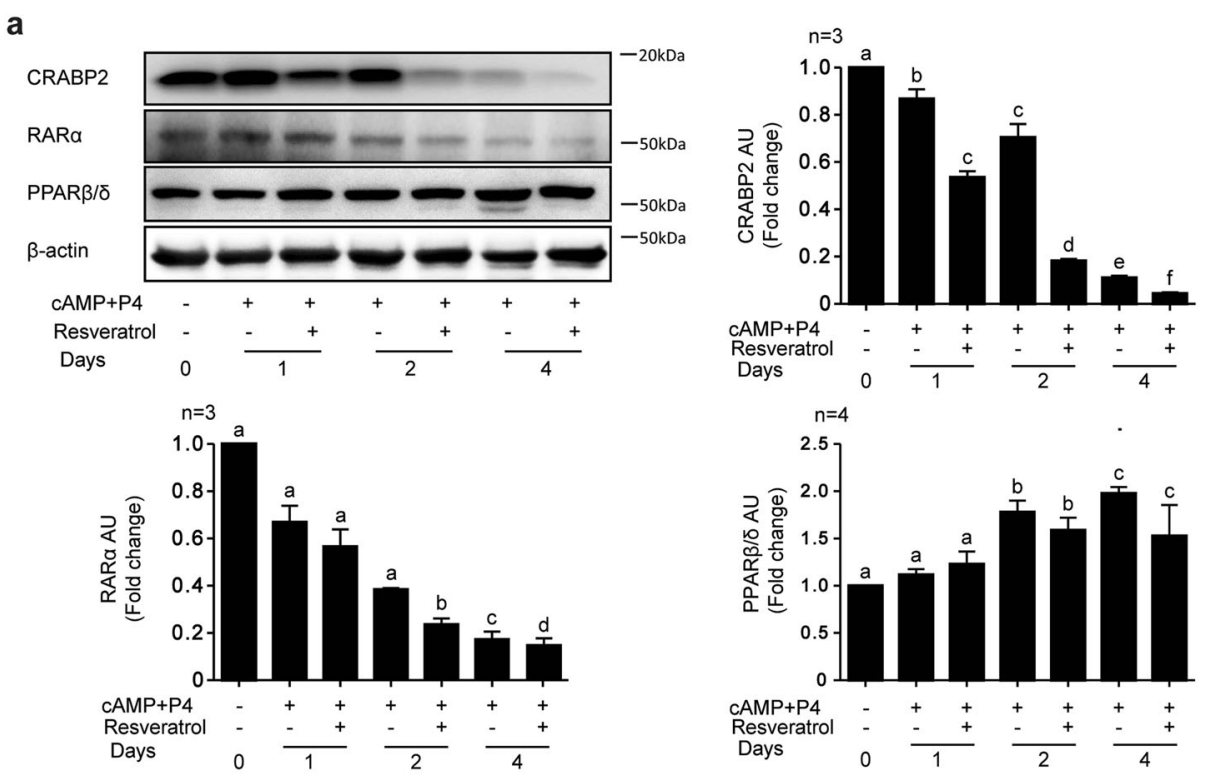

b
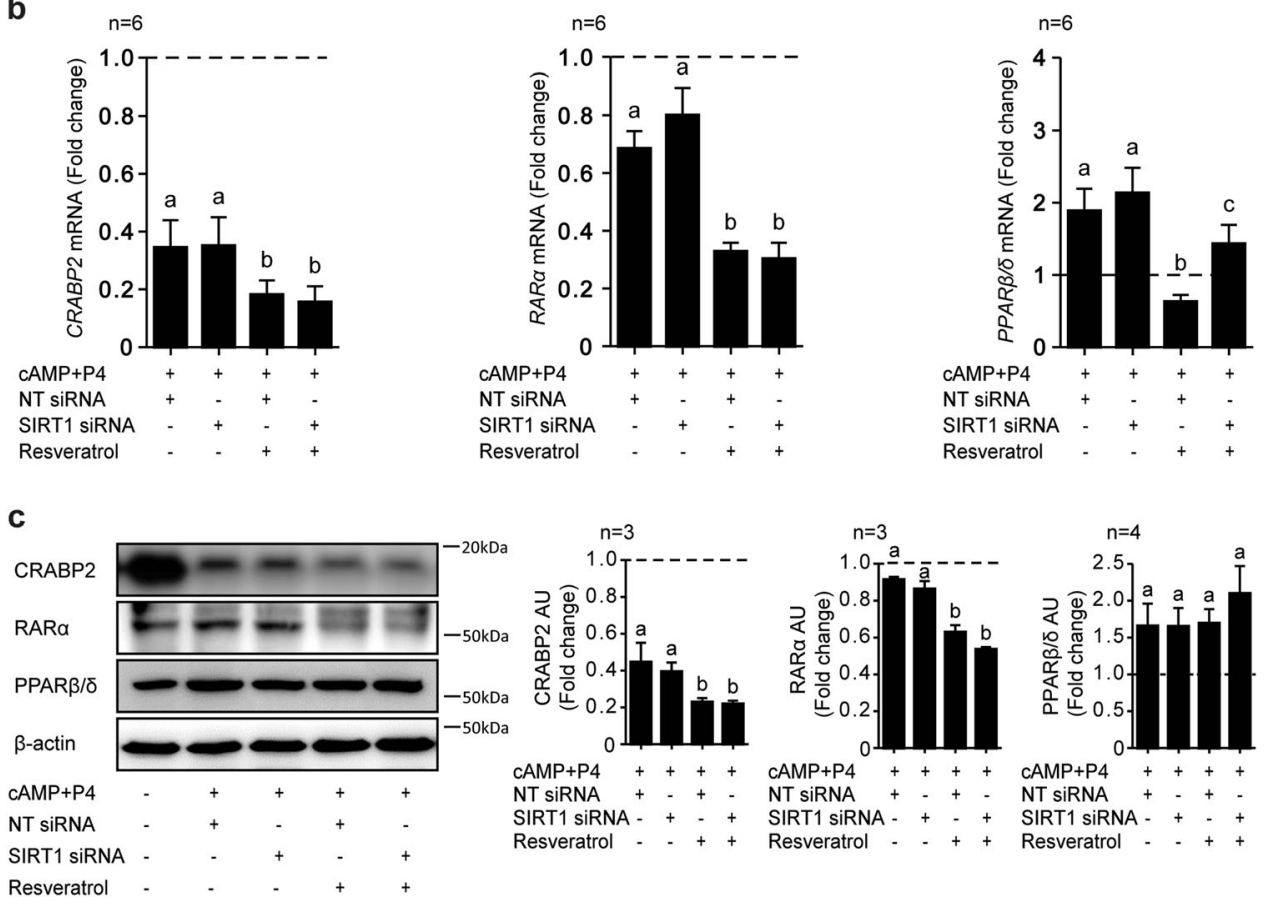

Fig. 4 Resveratrol advances the downregulation of the CRABP2-RARa pathway in decidualizing HESCs. a Representative Western blot and quantification of CRABP2, RARa, and PPARß/ $\delta$ proteins obtained from whole-cell lysates in undifferentiated or decidualized cells treated with cAMP and P4 in combination with or without resveratrol $(100 \mu \mathrm{M})$ for the indicated timepoints $(n=3)$. $\beta$-actin serves as a loading control. b RTQ-PCR analysis of CRABP2, RARa, and PPARB/ $\delta$ transcript levels in HESC cultures $(n=6)$ first transfected with NT or SIRT1 siRNA and then treated with cAMP and P4 in combination with or without resveratrol for 4 days. c Representative Western blot and quantification of RA related genes in whole cell lysates $(n=3)$ from parallel primary cultures. $\beta$-actin serves as a loading control. Data show fold-change (mean \pm SEM) relative to vehicle-treated undifferentiated cells (dotted line). Different letters above error bars denote significance at $P<0.05$

HESCs that failed to acquire a specialized phenotype and emerge as acutely senescent cells that secrete various proinflammatory cytokines, chemokines and proteases involved in tissue remodeling. Targeting and selective clearance of acutely senescent decidual cells by uNK cells terminates the inflammatory implantation window and effectively rejuvenates the endometrium at the time of embryo implantation ${ }^{15}$. An aberrant decidual response is 


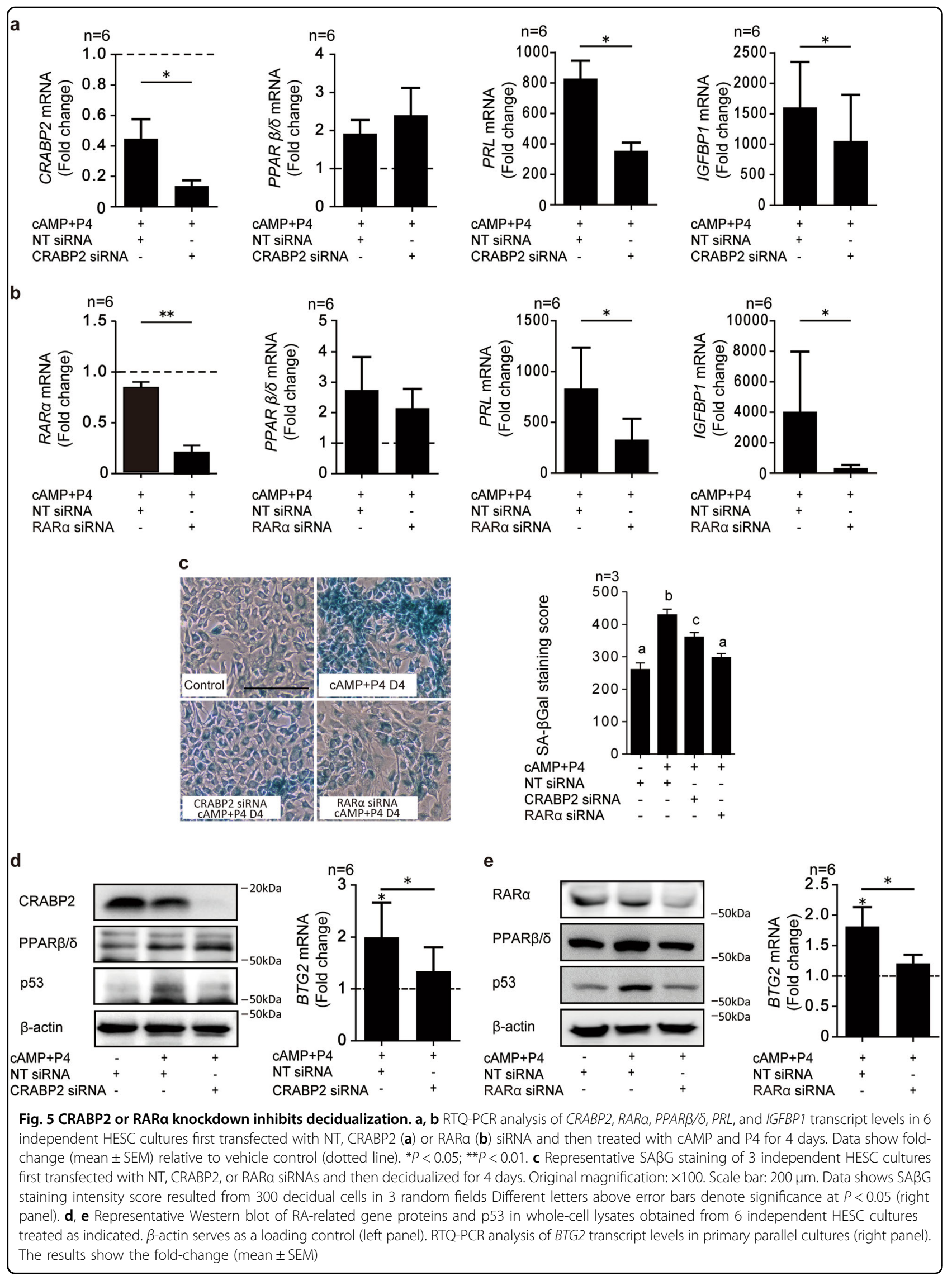


associated implantation failure, recurrent pregnancy loss and preeclampsia ${ }^{36-39}$. Preventing these prevalent reproductive disorders is a clinical priority but challenging because of a lack of effective therapeutic interventions.

Resveratrol is a widely used and well tolerated compound exhibiting anticancer ${ }^{40}$ immunomodulatory ${ }^{41}$ and antioxidant properties ${ }^{42,43}$. Although increasingly advocated for the treatment of reproductive failure associated with PCOS and obesity ${ }^{8-10,44}$, we demonstrate here that resveratrol is a potent inhibitor of the decidual pathway. We previously reported that differentiating HESCs downregulate CRABP2 and RAR, effectively silencing the pro-apoptotic arm of RA signaling, whilst simultaneously up-regulating the pro-differentiation RA-responsive nuclear receptor PPAR $\beta / \delta^{20}$. We now show that resveratrol both accelerates the downregulation of CRABP2 and $R A R \alpha$ and supresses PPAR $\beta / \delta$ induction. Knockdown experiments demonstrated that premature loss of CRABP2 or RAR $\alpha$ is sufficient to block transcriptional reprogramming of HESCs as well as the emergence of distinct decidual subpopulations. As a direct RAR target gene, BTG2 transcriptionally reduces cyclin D1 availability, inhibiting $G_{1}-S$ progression ${ }^{31,45,46}$. Exit from the cell cycle is a prerequisite for the emergence of terminally differentiated as well as senescent cells, and hence, indispensable for optimal decidualization of HESCs. We reported previously that cAMP-dependent downregulation of MDM2 proto-oncogene, an E3 ubiquitin ligase, stabilizes p53 in differentiating $\mathrm{HESCs}^{30}$. We now show that resveratrol prevents p53 accumulation in response to cAMP and P4 signaling in a SIRT-1 independent manner. It has previously been reported that resveratrol ameliorates age-related metabolic disorders by competitively inhibiting cAMP phosphodiesterases, resulting in enhanced cAMP levels ${ }^{47}$. Combined with a previous observation that p53 accumulates in resveratrol treated breast cancer cell lines ${ }^{48}$, our findings reinforce that resveratrol acts in both a tissue- and context-specific manner. Notably, p53 activates dehydrogenase/reductase 3 (DHRS3), a key enzyme in retinoid metabolism ${ }^{49,50}$. DHRS3 facilitates the reduction of retinal to retinol, and is induced upon decidualization ${ }^{21}$. As such, resveratrolmediated suppression of p53 is likely to perturb the cellular concentrations of bioactive retinoids.

Mature decidual cells are programmed to endure a range of environmental stressors, giving rise to a protective matrix able to withstand the intense tissue remodeling and vascular changes associated with implantation and placental formation. Coordinated silencing of multiple pathways including inositol triphosphate (IP3) signaling ${ }^{19}$, c-Jun $\mathrm{N}$-terminal kinase (JNK) signaling ${ }^{17}$ as well as cessation of endometrial circadian rhythms ${ }^{18}$ underpins this quasi-autonomous decidual state. Inhibition of the pro-apoptotic CRABP2-RAR arm of RA signaling renders the endometrium relatively resistant to the potentially adverse effects of RA. By contrast, upregulation of PPAR $\beta / \delta$ is a hallmark of implantation sites in the rat uterus ${ }^{51}$. Although. co-treatment of HESCs with a PPPAR $\beta / \delta$ agonist had no effect on the induction of $P R L$ or IGFBP1, the expression levels of both genes may already be maximal in cultures treated with cAMP and P4.

In summary, resveratrol is a promising therapeutic agent in reproductive medicine $e^{6,9,44,52}$, although its impact on embryo implantation or early pregnancy loss has not yet been evaluated. Pre-treatment of primary HESC cultures with resveratrol for $48 \mathrm{~h}$ prior to decidualization had no detrimental effects on the induction of $P R L$ and IGFBP1. The half-life of resveratrol is only $9-10 \mathrm{~h}$ in humans ${ }^{53,54}$. As such, limiting the use of resveratrol to the proliferative phase of the cycle may be the preferred strategy to improve ovarian function in selected patients without adversely impacting on subsequent embryo implantation, although this assertion requires further testing in clinical studies.

\section{Materials and methods \\ Patient selection}

This study was approved by the local ethical committee in Juntendo University, Faculty of Medicine (No. 14-103). Endometrial biopsies were obtained during the luteal phase of the cycle from patients without overt uterine pathology attending the Department of Obstetrics and Gynecology of Juntendo University Hospital, Tokyo, Japan. None of the participants were on hormonal therapy or taking resveratrol supplementation. Written, informed consent was obtained from all participating women prior to the biopsy.

\section{Primary culture of human endometrial stromal cells}

Collected HESCs were isolated, cultured, and maintained as described previously ${ }^{20,55}$. We obtained the endometrial samples in Dulbecco's modified Eagle's medium-Ham's (DMEM) F-12 (Nacalai Tesque, Kyoto, Japan) containing $1 \%(\mathrm{v} / \mathrm{v})$ antibiotic solution, minced finely and digested enzymatically with $5 \mathrm{mg}$ collagenase $(5 \mathrm{mg} / 100 \mu \mathrm{l})$ (Sigma-Aldrich, Saint Louis, USA) and deoxyribonuclease (DNase) type I $(100 \mu \mathrm{g} / \mu \mathrm{l})$ (Roche Applied Science, Mannheim, Germany) for $1 \mathrm{~h}$ at $37^{\circ} \mathrm{C}$. After centrifugation, the cells were suspended in DMEM/ F12 culture media containing 10\% (v/v) dextran-coated charcoal (DCC) treated fetal bovine serum, 1\% antibiotic solution, $1 \%(\mathrm{v} / \mathrm{v})$ L-glutamine, $0.2 \%(\mathrm{v} / \mathrm{v})$ insulin and 1 $\mathrm{nM}$ estradiol (all Sigma-Aldrich). Endometrial cells were cultured until confluence in $75 \mathrm{~cm}^{2}$ culture flasks at $37^{\circ} \mathrm{C}$ in $5 \%$ carbon dioxide and then passaged once or twice. Cells were discarded after passage 3. In decidualization experiments, confluent monolayers were maintained in DMEM/F12 without phenol red (GIBCO, life 
technologies, Grand Island, USA) containing 2\% (v/v) DCC-FBS and treated with $0.5 \mathrm{mM} 8$-bromo-cAMP, $1 \mu \mathrm{M}$ $\mathrm{P} 4$ in combination with or without $100 \mu \mathrm{M}$ resveratrol (all Sigma-Aldrich).

\section{Immunohistochemical staining}

Confluent HESC cultures in 4-well chamber slides were decidualized as described above in the presence or absence of $100 \mu \mathrm{M}$ resveratrol for 4 or 8 days. Following treatment, cells were fixed with $4 \%$ buffered paraformaldehyde, endogenous peroxidase activity blocked with $1 \% \mathrm{H}_{2} \mathrm{O}_{2}$ and probed with anti-SIRT1 antibody at a 1:100 dilution overnight (Abcam, Cambridge, UK). Excess primary antibody was washed with TBS-T and probed with a secondary antirabbit antibody at a 1:300 dilution (DAKO, Glostrup, Denmark). The expression of SIRT1 was detected using horseradish peroxidase-conjugated streptavidin, 1:300 (DAKO) and visualized with SIGMAFAST ${ }^{\mathrm{m}}$ DAB with Metal Enhancer (Sigma-Aldrich), in which Cobalt chloride was added to enhance 3,3'-diaminobenzidine reaction. Eosin was used as a cytoplasmic counterstain. Images were taken using Keyence BZ-X700 microscope with 40×, 100×, or 200× magnifications (Keyence, Osaka, Japan).

\section{RTQ-PCR}

Total RNA was extracted from primary HESC cultures using RNeasy plus mini kit (QIAGEN, Hilden, Germany). We generated cDNA with the SuperScript II Reverse Transcriptase for RT-PCR kit (Invirtrogen Ltd. Life technology, Carlsbad, USA) and performed Template quantification using 7500 fast real-time PCR system (Applied Biosystems, Forster City, USA) with dye layer, power SYBER Green PCR Master Mix (Applied Biosystems). RNA input variances were normalized against the levels of housekeeping gene, L19 which encodes a ribosomal protein. All measurements were performed in duplicate or triplicate. The gene-specific primer pairs were designed by using Primer3 software (http://frodo.wi. mit.edu): L19 sense, $5^{\prime}$-GCG GAA GGG TAC AGC CAA T-3', L19 antisense, 5'-GCA GCC GGC GCA AA-3'; SIRT1 sense $5^{\prime}$-TCT AAC TGG AGC TGG GGT G-3' and SIRT1 antisense, $5^{\prime}$-AAG TCT ACA GCA AGG CGA GC-3'; decidual $P R L$ sense $5^{\prime}$-AAG CTG TAG AGA TTG AGG AGC AAA C-3' and decidual PRL antisense, 5'TCA GGA TGA ACC TGG CTG ACT A-3'; IGF-binding protein 1 (IGFBP1) sense, 5' -CGA AGG CTC TCC ATG TCA CCA-3' and IGFBP1 antisense, 5'-TGT CTC CTG TGC CTT GGC TAA AC-3'; CRABP2 sense, 5'-TGT GAG CAG AAG CTC CTG AAG-3'and CRABP2 antisense 5'-GTT CTA CCT GTG GCC ACT CAC T-3'; $R A R \alpha$ sense, 5'-GCC CAG CTC ACC ACA TCT TC-3' and $R A R \alpha$ antisense, $5^{\prime}$-GGA GCA ATG GCT TGT GAG TTCT-3'; $P P A R \beta / \delta$ sense, $5^{\prime}$-ACT GAC CCA ACT GAT CCT GCT C-3' and PPAR $\beta / \delta$ antisense, $5^{\prime}$-GCC TGG
CAA ACC AGT GTG AA-3'; BTG2 sense, 5' -ACG GGA AGG GAA CCG ACA T-3' and BTG2 antisense 5'-CAG TGG TGT TTG TAG TGC TCT G-3'. Proprietary commercially available primers for FABP5 (QIAGEN, Maryland, USA) were used.

\section{Western blot analysis}

Total protein lysates were extracted from HESCs by direct lysis in SDS sample buffer heated to $75^{\circ} \mathrm{C}$. Proteins resolved by SDS-PAGE were separated in a polyacrylamide gel (Bio-rad laboratories, Hercules, USA) and transferred to Immobilon-P membrane (Merck, Darmstadt, Germany) and probed with antibodies raised against SIRT1, 1:500 (Abcam) CRABP2, 1:5000 (Sigma-Aldrich); FABP5, 1:1000 (Abcam); RAR $\alpha, 1: 5000$ (Abcam); PPAR $\beta /$ $\delta, ~ 1: 1000$ (Abcam); p53, 1:1000 (Cell Signaling Technology, Danvers, USA), and $\beta$-actin, 1:5000 (Sigma-Aldrich). After incubation with peroxidase-conjugated secondary antibodies, (Jackson Immunoresearch Laboratories, West Grobe, USA), detection of chemiluminescence was visualized with Pierce Western Blotting Substrate Plus (Thermo Fisher Scientific, Rockford, USA) or Super Signal West Dura Extended Duration Substrate (Thermo Fisher Scientific) and normalized to $\beta$-actin. Western blots were quantified by densitometry using Multi Gauge software Version3.0 (Fuji Photo Film, Tokyo, Japan).

\section{RNA interference}

Primary HESCs were seeded in six-well plates and cultured to $70 \%$ confluence for $24 \mathrm{~h}$. Cells were then transfected with siRNAs against genes encoding SIRT1 (M003540-0010, siGENOME SMARTpool siRNA, Dharmacon, MA, USA), CRABP2 (M-003448-01, siGENOME SMARTpool siRNA, Dharmacon), RAR (M-003437-02, siGENOME SMARTpool siRNA, Dharmacon), or negative control siRNA (452001, Stealth RNAi Negative Control, invitrogen, Carlsbad, USA) using Lipofectamine RNAiMAX Reagent (invitrogen, Carlsbad, USA), according to the manufacturer's instruction. For transfection, 25 pmol of siRNAs and $5 \mu \mathrm{l}$ of Lipofectamine RNAiMAX were diluted with $500 \mu \mathrm{l}$ of reduced serum medium (Opti-MEM, Invitrogen, Life Technologies) and mixed. The transfection mixture was incubated for $20 \mathrm{~min}$ at room temperature and added dropwise to each well containing $2 \mathrm{ml}$ of DMEM/F12 with 5\% (v/v) DCC-FBS (final siRNA concentration: 10 $\mathrm{nM})$. Twenty-four hours following transfection, cells were washed with PBS and decidualized as described above.

\section{SABG staining}

Following 4 or 8 days of decidualization, cells were stained with X-gal using Senescence $\beta$-Galactosidase Staining Kit (Cell Signaling Technology, Danvers, USA) according to manufacturer's instruction. After $24 \mathrm{~h}$ of incubation, cells were observed using a phase-contrast 
microscopy. Images were taken using Keyence BZ-X710 microscope with $40 \times, 100 \times$, or $200 \times$ magnifications (Keyence, Osaka, Japan). Senescent cells were detected as blue-stained cells, the staining intensity of positive cells were scored as 0 (absent staining); 1 (partial cytoplasmic staining); 2 (total cytoplasmic staining). A total of 300 cells were counted in three random fields on a culture plate for each sample (Supplementary Figure S4).

\section{Statistical analysis}

Data are representative of three or more biological replicates. Results are reported as mean \pm standard error (SEM). Statistical analysis was performed using one-way analysis of variance (ANOVA) followed by Wilcoxon signed rank test or Mann-Whitney test within groups, following normalization of the data with GraphPad Prism 5 (GraphPad Software Inc., San Diego, USA). The level of significance was defined as $P$ value of $<0.05$.

\section{Acknowledgements}

We wish to thank all participating women. We also thank Dr. Shinji Nakamura in Division of Biomedical Imaging Research, Juntendo University Graduate School of Medicine for technical assistant of Immunohistochemical staining, Dr. Reiko Mineki in Division of Proteomics and Biomolecular Science, Biomedical Research Center Laboratory of Molecular and Biochemical Research, Juntendo University Graduate School of Medicine for technical assistant of Western blot analysis, Research Support Center, Juntendo University Graduate School of Medicine, Tokyo, Japan, for technical assistance. K.K. was supported by JSPS KAKENHI Grant Number 18K09273 and the Medical Research Encouragement Prize of the Japan Medical Association. K.M. was supported by JSPS KAKENHI Grant Number 16K20208. J.J.B. was supported by the Wellcome Trust (2/2233/z/18/2) and the Tommy's baby charity, United Kingdom.

\section{Author details}

${ }^{1}$ Department of Obstetrics and Gynaecology, Faculty of Medicine, Juntendo University, Tokyo 113-8421, Japan. ${ }^{2}$ Center for Reproductive Medicine and Implantation Research, Sugiyama Clinic Shinjuku, Tokyo 116-0023, Japan. ${ }^{3}$ The Division of Biomedical Sciences, Clinical Science Research Laboratories, Warwick Medical School, Coventry CV2 2DX, UK. ${ }^{4}$ Tommy's National Centre for Miscarriage Research, University Hospitals Coventry \& Warwickshire, Coventry CV2 2DX, UK

\section{Conflict of interest}

The authors declare that they have no conflict of interest.

\section{Publisher's note}

Springer Nature remains neutral with regard to jurisdictional claims in published maps and institutional affiliations.

Supplementary Information accompanies this paper at (https://doi.org/ 10.1038/s41419-019-1511-7).

Received: 19 October 2018 Revised: 19 February 2019 Accepted: 7 March 2019

Published online: 20 March 2019

\section{References}

1. Pirola, L. \& Fröjdö, S. Resveratrol: one molecule, many targets. IUBMB Life $\mathbf{6 0}$, 323-332 (2008).

2. Diaz-Gerevini, G. T. et al. Beneficial action of resveratrol: How and why? Nutrition 32, 174-178 (2016).
3. Houtkooper, R. H., Pirinen, E. \& Auwerx, J. Sirtuins as regulators of metabolism and healthspan. Nat. Rev. Mol. Cell Biol. 13, 225-238 (2012).

4. Imai, S., Armstrong, C. M., Kaeberlein, M. \& Guarente, L. Transcriptional silencing and longevity protein Sir2 is an NAD-dependent histone deacetylase. Nature 403, 795-800 (2000).

5. Chung, S. et al. Regulation of SIRT1 in cellular functions: role of polyphenols. Arch. Biochem. Biophys. 501, 79-90 (2010).

6. Liu, M. et al. Resveratrol protects against age-associated infertility in mice. Hum. Reprod. 28, 707-717 (2013).

7. Zhang, T. et al. SIRT1, 2, 3 protect mouse oocytes from postovulatory aging. Aging 8, 685-696 (2016).

8. Cabello, E. et al. Effects of resveratrol on ovarian response to controlled ovarian hyperstimulation in ob/ob mice. Fertil. Steril. 103, 570-579.e571 (2015).

9. Ortega, I. \& Duleba, A. J. Ovarian actions of resveratrol. Ann. N. Y. Acad. Sci. 1348, 86-96 (2015)

10. Aquino, C. I. \& Nori, S. L. Complementary therapy in polycystic ovary syndrome. Transl. Med. @ Unisa 9, 56-65 (2014).

11. Juan, M. E. et al. Trans-resveratrol, a natural antioxidant from grapes, increases sperm output in healthy rats. J. Nutr. 135, 757-760 (2005).

12. Kolahdouz Mohammadi, R. \& Arablou, T. Resveratrol and endometriosis: in vitro and animal studies and underlying mechanisms (Review). Biomed. Pharmacother. 91, 220-228 (2017).

13. Ho, Y. et al. Resveratrol inhibits human leiomyoma cell proliferation via crosstalk between integrin alphavbeta3 and IGF-1R. Food Chem. Toxicol. 120, 346-355 (2018)

14. Gellersen, B. \& Brosens, J. J. Cyclic decidualization of the human endometrium in reproductive health and failure. Endocr. Rev. 35, 851-905 (2014).

15. Brighton, P. J. et al. Clearance of senescent decidual cells by uterine natural killer cells in cycling human endometrium. Elife 6, e31274 (2017).

16. Cloke, B. et al. The androgen and progesterone receptors regulate distinct gene networks and cellular functions in decidualizing endometrium. Endocrinology 149, 4462-4474 (2008).

17. Leitao, B. et al. Silencing of the JNK pathway maintains progesterone receptor activity in decidualizing human endometrial stromal cells exposed to oxidative stress signals. FASEB J. 24, 1541-1551 (2010).

18. Muter, J. et al. The clock protein period 2 synchronizes mitotic expansion and decidual transformation of human endometrial stromal cells. FASEB J. 29, 1603-1614 (2015).

19. Muter, J. et al. Progesterone-dependent induction of phospholipase C-related catalytically inactive protein 1 (PRIP-1) in decidualizing human endometrial stromal cells. Endocrinology 157, 2883-2893 (2016).

20. Ozaki, R. et al. Reprogramming of the retinoic acid pathway in decidualizing human endometrial stromal cells. PLOS ONE 12, e0173035 (2017).

21. Kuroda, K. et al. Induction of 11ß-HSD 1 and activation of distinct mineralocorticoid receptor- and glucocorticoid receptor-dependent gene networks in decidualizing human endometrial stromal cells. Mol. Endocrinol. 27, 192-202 (2013)

22. Noy, N. Non-classical transcriptional activity of retinoic acid. Subcell. Biochem 81, 179-199 (2016)

23. Schug, T. T., Berry, D. C., Shaw, N. S., Travis, S. N. \& Noy, N. Opposing effects of retinoic acid on cell growth result from alternate activation of two different nuclear receptors. Cell 129, 723-733 (2007).

24. Bastien, J. \& Rochette-Egly, C. Nuclear retinoid receptors and the transcription of retinoid-target genes. Gene 328, 1-16 (2004).

25. Tang, S. et al. SIRT1-mediated deacetylation of CRABPII regulates cellular retinoic acid signaling and modulates embryonic stem cell differentiation. $\mathrm{Mol}$. Cell 55, 843-855 (2014).

26. Kang, M. R. et al. Reciprocal roles of SIRT1 and SKIP in the regulation of RAR activity: implication in the retinoic acid-induced neuronal differentiation of P19 cells. Nucleic Acids Res. 38, 822-831 (2010).

27. Yu, S., Levi, L., Siegel, R. \& Noy, N. Retinoic acid induces neurogenesis by activating both retinoic acid receptors (RARs) and peroxisome proliferator-activated receptor $\beta / \delta$ (PPARß/§). J. Biol. Chem. 287, 42195-42205 (2012).

28. Leno-Durán, E. et al. Human decidual stromal cells secrete soluble proapoptotic factors during decidualization in a CAMP-dependent manner. Hum. Reprod. 29, 2269-2277 (2014).

29. Dimri, G. P. et al. A biomarker that identifies senescent human cells in culture and in aging skin in vivo. Proc. Natl Acad. Sci. USA 92, 9363-9367 (1995). 
30. Pohnke, Y. et al. Wild-type p53 protein is up-regulated upon cyclic adenosine monophosphate-induced differentiation of human endometrial stromal cells. J. Clin. Endocrinol. Metab. 89, 5233-5244 (2004).

31. Donato, L. J., Suh, J. H. \& Noy, N. Suppression of mammary carcinoma cell growth by retinoic acid: the cell cycle control gene Btg2 is a direct target for retinoic acid receptor signaling. Cancer Res. 67, 609-615 (2007).

32. Tirone, F. The gene PC3 (TIS21/BTG2), prototype member of the PC3/BTG/TOB family: regulator in control of cell growth, differentiation, and DNA repair? J. Cell. Physiol. 187, 155-165 (2001)

33. Qin, S., Lu, Y. \& Rodrigues, G. A. Resveratrol protects RPE cells from sodium iodate by modulating PPARa and PPARS. Exp. Eye Res. 118, 100-108 (2014).

34. Paria, B. C. et al. Coordination of differential effects of primary estrogen and catecholestrogen on two distinct targets mediates embryo implantation in the mouse. Endocrinology 139, 5235-5524 (1998).

35. Kin, K. et al. The transcriptomic evolution of mammalian pregnancy: gene expression innovations in endometrial stromal fibroblasts. Genome Biol. Evol. 8, 2459-2473 (2016).

36. Karpovich, N. et al. The production of interleukin-11 and decidualization are compromised in endometrial stromal cells derived from patients with infertility. J. Clin. Endocrinol. Metab. 90, 1607-1612 (2005).

37. Salker, M. S. et al. Deregulation of the serum-and glucocorticoid-inducible kinase SGK1 in the endometrium causes reproductive failure. Nat. Med. 17 1509 (2011).

38. Salker, M. S. et al. Disordered IL-33/ST2 activation in decidualizing stromal cells prolongs uterine receptivity in women with recurrent pregnancy loss. PLoS ONE 7, e52252 (2012).

39. Piltonen, T. T. et al. Endometrial stromal fibroblasts from women with polycystic ovary syndrome have impaired progesterone-mediated decidualization, aberrant cytokine profiles and promote enhanced immune cell migration in vitro. Hum. Reprod. 30, 1203-1215 (2015).

40. Varoni, E. M., Lo Faro, A. F., Sharifi-Rad, J. \& Iriti, M. Anticancer molecular mechanisms of resveratrol. Front. Nutr. 3, 8 (2016).

41. Salehi, B. et al. Resveratrol: a double-edged sword in health benefits. Biomedicines 6, 91 (2018).

42. Konyalioglu, S., Armagan, G., Yalcin, A., Atalayin, C. \& Dagci, T. Effects of resveratrol on hydrogen peroxide-induced oxidative stress in embryonic neural stem cells. Neural Regen. Res. 8, 485 (2013).
43. Means, J. C., Gerdes, B. C. \& Koulen, P. Distinct mechanisms underlying resveratrol-mediated protection from types of cellular stress in C6 glioma cells. Int. J. Mol. Sci. 18, 1521 (2017).

44. Banaszewska, B., Wrotyńska-Barczyńska, J., Spaczynski, R. Z., Pawelczyk, L. \& Duleba, A. J. Effects of resveratrol on polycystic ovary syndrome: a doubleblind, randomized, placebo-controlled trial. J. Clin. Endocrinol. Metab. 101 4322-4328 (2016).

45. Guardavaccaro, D. et al. Arrest of G(1)-S progression by the $\mathrm{p} 53$-inducible gene PC3 is Rb dependent and relies on the inhibition of cyclin D1 transcription. Mol. Cell. Biol. 20, 1797-1815 (2000).

46. Lim, I. K. TIS21 (/BTG2/PC3) as a link between ageing and cancer: cell cycle regulator and endogenous cell death molecule. J. Cancer Res. Clin. Oncol. 132, 417-426 (2006)

47. Park, S.-J. et al. Resveratrol ameliorates aging-related metabolic phenotypes by inhibiting CAMP phosphodiesterases. Cell 148, 421-433 (2012).

48. Kim, Y. et al. Resveratrol inhibits cell proliferation and induces apoptosis of human breast carcinoma MCF-7 cells. Oncol. Rep. 11, 441-446 (2004).

49. Kirschner, R. D., Rother, K. Müller, G. A. \& Engeland, K. The retinal dehydrogenase/reductase retSDR1/DHRS3 gene is activated by p53 and p63 but not by mutants derived from tumors or EEC/ADULT malformation syndromes. Cell Cycle 9, 2177-2188 (2010).

50. Deisenroth, C., Itahana, Y., Tollini, L., Jin, A. \& Zhang, Y. p53-Inducible DHRS3 is an endoplasmic reticulum protein associated with lipid droplet accumulation. J. Biol. Chem. 286, 28343-28356 (2011).

51. Ding, N. Z., Ma, X. H., Diao, H. L., Xu, L. B. \& Yang, Z. M. Differential expression of peroxisome proliferator-activated receptor delta at implantation sites and in decidual cells of rat uterus. Reproduction 125, 817-825 (2003).

52. Tatone, $\mathrm{C}$. et al. Sirtuins in gamete biology and reproductive physiology: emerging roles and therapeutic potential in female and male infertility. Hum. Reprod. Update 24, 267-289 (2018).

53. Goldberg, D. M., Yan, J. \& Soleas, G. J. Absorption of three wine-related polyphenols in three different matrices by healthy subjects. Clin. Biochem. 36, 79-87 (2003).

54. Gescher, A. J. \& Steward, W. P. Relationship between mechanisms, bioavailibility, and preclinical chemopreventive efficacy of resveratrol: a conundrum. Cancer Epidemiol. Biomark. Prev. 12, 953-957 (2003).

55. Barros, F. S., Brosens, J. J. \& Brighton, P. J. Isolation and primary culture of various cell types from whole human endometrial biopsies. Bio-Protocol 6, e2028 (2016) 